\title{
Analytical Method for 3-D Stopping Sight Distance Adequacy Investigation
}

\author{
Fotis S. Mertzanis ${ }^{1}$, Antonis Boutsakis ${ }^{1}$, Ikaros-Georgios Kaparakis ${ }^{1}$, Stergios Mavromatis ${ }^{2}$ and Basil Psarianos ${ }^{3}$ \\ 1. School of Civil Engineering, National Technical University of Athens, Athens 15452, Greece \\ 2. School of Civil Engineering and Surveying and Geoinformatics Engineering, Technological Educational Institute of Athens,
} Athens 12210, Greece

3. School of Rural and Surveying Engineering, National Technical University of Athens, Athens 15452, Greece

\begin{abstract}
The adopted 2-D SSD (stopping sight distance) adequacy investigation in current design practice may lead to design deficiencies due to inaccurate calculation of the available sight distance. Although this concern has been identified by many research studies in the past, none of them suggested a comprehensive methodology to simulate from a 3-D perspective concurrently both the cross-section design and the vehicle dynamics in space during emergency braking conditions. The proposed methodology can accurately perform SSD adequacy investigation in any 3-D road environment where the ground, road and roadside elements are inserted by identifying areas of interrupted vision lines between driver and obstacle being less than the required distance necessary to bring the vehicle to a stop condition. The present approach provides flexibility among every road design and/or vehicle dynamic parameter inserted, as well as direct overview regarding design elements that restrict the driver's vision and create SSD inadequacies. As a result, precious guidance is provided to the designer for further alignment improvement but mostly an accurate aid to implement geometric design control criteria with respect to both existing as well as new road sections is delivered. The efficiency of the suggested methodology is demonstrated through a case study.
\end{abstract}

Key words: SSD, 3-D road alignment, design consistency, road safety.

\section{Introduction}

In existing road design policies [1-4], despite the fact that $3-\mathrm{D}$ perspective is essential in order to evaluate the final outcome, the adoption of critical design parameters is restricted on a fragmented 2-D road environment. Such a typical case of design misconception is performed while determining the critical parameter of SSD (stopping sight distance).

The 2-D SSD calculation is inaccurate. The impact of this approach can be detrimental to the cost and/or design consistency of existing and new road facilities, in terms of adopting excessive overdesign suggestions (e.g., increase of the inner shoulder width of divided highways) or unnecessary posted speed areas, where, in each case, safety violations might occur as well.

Corresponding author: Fotis S. Mertzanis, Ph.D. candidate, research field: transportation engineering. E-mail: fmertz@otenet.gr.
In current practice, some efforts have been made recently to overcome this incorrect SSD determination by establishing some coordination between the horizontal and vertical curve positioning, although not all the design cases are addressed. For example, the Green Book [1] stresses that in order to grant the SSD provision, the vertical transition curve should be entirely designed inside the horizontal curve. In the relevant Spanish Design Guidelines [3], the desired horizontal-vertical arrangement is reached when the vertical crest curve falls completely inside the horizontal curve including spirals.

The present paper introduces an analytical method for 3-D sight distance analysis that considers the 3-D configuration of the roadway as well as the dynamics of a vehicle moving along the actual 3-D roadway path, based on the difference between the available and the demanded SSD. 
The suggested SSD adequacy investigation is incorporated in "H12" (2012) road design software, developed at the NTUA (National Technical University of Athens) for academic purposes [5], where many consultant firms in Greece use it as well.

The efficiency of the suggested methodology is demonstrated through a case study.

\section{SSD Modeling}

The SSD adequacy analysis is based on either 2-D or 3-D models. The 2-D SSD investigation is rather fragmentary and may produce design deficiencies due to inaccurate calculation of the available sight distance, where even more critical situations might occur. Hassan et al. [6], for example, stated that 2-D SSD investigation may underestimate or overestimate the available sight distance and consequently lead to safety violation.

This concern has been identified by many researchers in the past and a wide range of 2-D and 3-D approaches have been developed to address the problem. One of the first researchers that assessed the available sight distance on 3-D alignment, Sanchez [7] studied the interaction between the sight distance and the 3-D combined alignment idealized into a net of triangles using inroads software. In this research, the operator, assisted by the different views generated by the computer, was able to determine the obstruction impeding the driver's sight line. Although this methodology was accurate, it was very time consuming since the available sight distance was determined graphically (not analytically).

Several years later, Hassan et al. [8] presented an analytical model for computing available sight distance on combined horizontal and vertical highway alignments, using parametric finite elements (four, six and eight-node rectangular elements as well as three-node triangular elements) to represent the highway and sight obstructions. The idea behind the proposed model is summarized in checking the driver's sight line, which is represented by a straight line between the driver's eye and an object, against all the possible sight obstructions, by using an iterative procedure.

Lovell et al. [9] developed a method to calculate the sight distance based on horizontal geometry, without considering the effect of vertical geometry. Nehate and Rys [10] described a methodology to define the available sight distance using GPS (global positioning system) data by examining the intersection of line of sight with the elements representing the road surface. However, the available sight distance was not based on the road's compound (horizontal and vertical) alignment.

In the past years, in order to evaluate the actual sight distance in real driving conditions, a number of 3-D models are found in the literatures [11-17] which were based on their performance through the correlation between the road surface, the ground terrain and the roadside environment aiming to optimize the available sight distance.

Recently, Kim and Lovell [18] delivered a 3-D sight distance evaluation method where an algorithm is used to determine the maximum available sight distance using computational geometry and thin plate spline interpolation to represent the surface of the road. The available sight distance is measured by finding the shortest line that does not intersect any obstacle.

Jha et al. [19] proposed a similar to the present paper 3-D methodology for measuring sight distance along a roadway's centerline, utilizing triangulation methods via an introduced for this purpose algorithm, consisting of three stages, namely, road surface development, virtual field of view surface development, and virtual line of sight plane development. However, the process involved multiple software platforms, thus delivering an accurate but non-flexible outcome.

The abovementioned 3-D models are capable of accurately simulating compound road environments where an unsuccessful arrangement of vertical and horizontal alignment may exist, and thus allows the definition of the actual vision field to the driver. 
However, as already stated above, most of the previously mentioned research studies are focused on optimizing the available SSD by introducing either new algorithms or design parameter combinations, ignoring, in many cases, the topographic visual restraints. Moreover, none of the abovementioned approaches suggested a comprehensive methodology to simulate from a 3-D perspective concurrently both the cross-section design and the vehicle dynamics in space during emergency braking conditions. Furthermore, design elements responsible for SSD inadequacies are accurately delivered, thus providing precious guidance to the designer for further alignment improvement.

\section{Methodology}

As to investigate concurrently vehicles dynamics and 3-D road perspective impacts in SSD evaluation, a "sub-software" has been developed at NTUA by the leading co-author [20] based on Microsoft's Excel environment (Visual Basic). This "sub-software" is part of H12 (2012) road design software [5] and the flowchart is shown in Fig. 1. H12 software consists of various individual sub-routines through which certain road design stages are processed, namely, the creation of terrain model, horizontal alignment, vertical alignment, cross-sections, Bruckner diagram and 3-D road model. Each sub-routine runs data entered by the user and delivers data outputs, accessible by notepad as well, and drawing outputs (in .dxf format).

The design of the road's typical cross-section, for example, is formed by the following distinctive parts: main cross-section, road formations, cut-fill slopes, and ground formations until the ground is reached (Fig. 2).

The SSD adequacy investigation in this paper is based on a recently developed methodology by the authors' process that relates the 3-D configuration of a roadway to the dynamics of a vehicle moving along the actual roadway path, based on the difference between

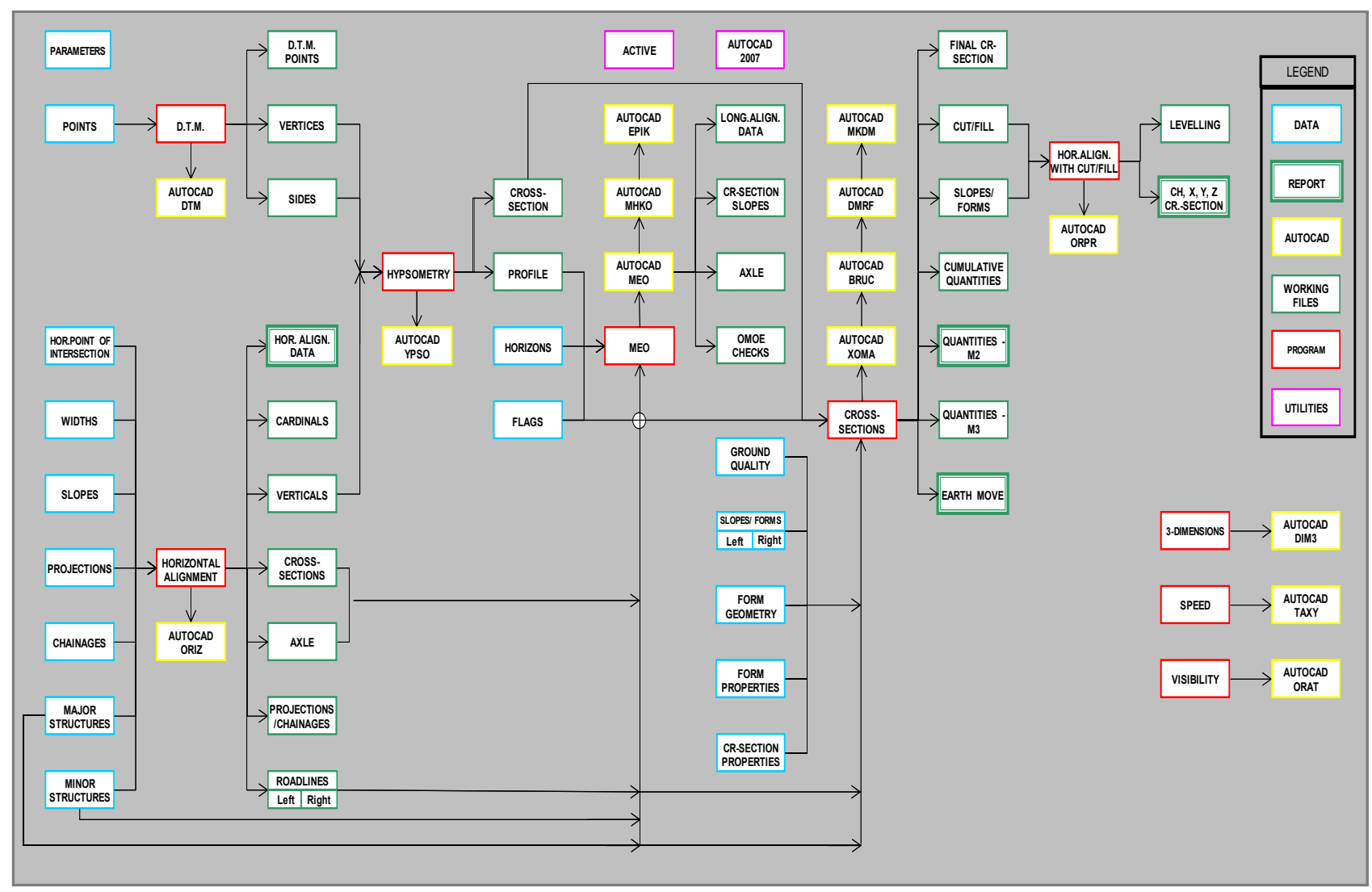

Fig. 1 H12 road design software interface. 


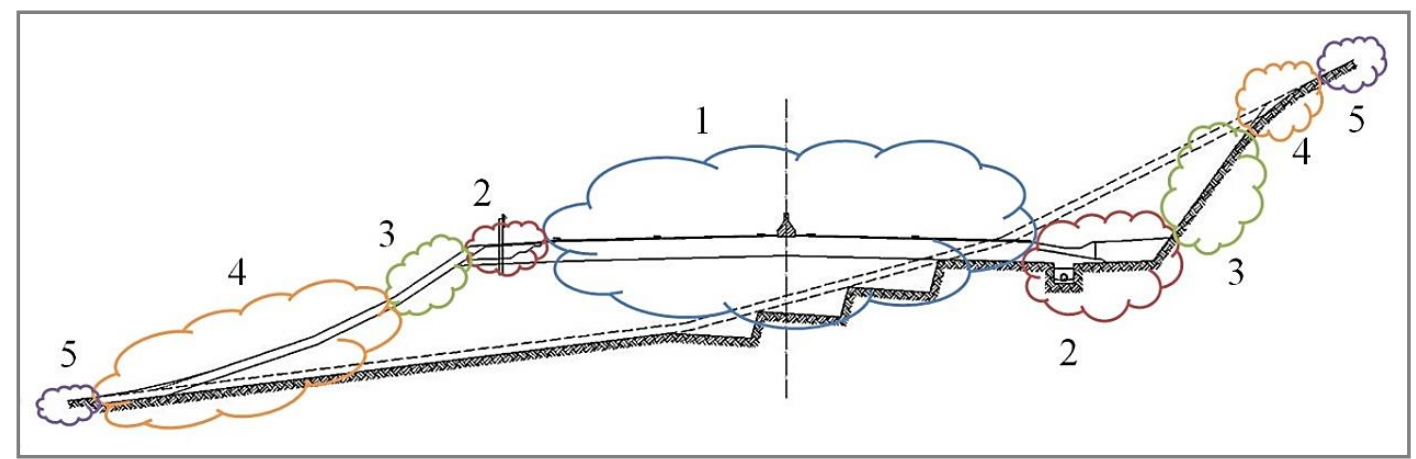

Fig. 2 Distinctive parts of a typical cross-section (divided or undivided roadway can be outlined (divided case shown): 1. main cross-section; 2. road formations; 3. cut-fillslopes; 4. ground formations; 5. ground).

the provided and the demanded SSD. Both $S S D_{\text {demanded }}$ and $S S D_{\text {available }}$ are briefly presented below.

\subsection{SSD Demanded Calculation}

According to existing design policies, the demanded SSD consists of two distance components: the distance traveled during driver's perception-reaction time to the instant, the brakes are applied and the distance while braking to stop the vehicle. For example, the SSD model adopted by many design policies is represented by Eq. (1):

$$
S S D=V_{0} t+\frac{V_{0}^{2}}{2 \mathrm{~g}\left(\frac{a}{\mathrm{~g}}+s\right)}
$$

where:

$V_{0}(\mathrm{~m} / \mathrm{s})$ : vehicle initial speed;

$t$ (s): driver's perception-reaction time (e.g., $2.5 \mathrm{~s}$ [1] and $2.0 \mathrm{~s}[2])$;

$\mathrm{g}\left(\mathrm{m} / \mathrm{s}^{2}\right)$ : gravitational constant;

$a\left(\mathrm{~m} / \mathrm{s}^{2}\right)$ : vehicle deceleration rate (e.g., $3.4 \mathrm{~m} / \mathrm{s}^{2}[1]$ and $\left.3.7 \mathrm{~m} / \mathrm{s}^{2}[2]\right)$;

$s(\% / 100)$ : road grade (upgrades $(+)$, downgrades $(-))$.

However, the above approach ignores curved areas of both horizontal and vertical alignment, since, on one hand, the portion of friction provided in the longitudinal direction, assigned to serve the braking process, is associated directly to the friction demanded laterally [21], and on the other hand, the grade values involved in vertical curves are variable. In order to incorporate the effect of these parameters, simple considerations based on the mass point model as well as the laws of mechanics were applied respectively.

Assuming the well-known Krempel's [21] friction circle, the actual longitudinal friction provided for braking on curved sections is expressed by Eq. (2):

$$
f_{T}=\sqrt{\left(\frac{a}{\mathrm{~g}}\right)^{2}-\left(\frac{V^{2}}{\mathrm{~g} R}-e\right)^{2}}
$$

where:

$f_{T}$ : friction demand in the longitudinal direction of travel;

$V(\mathrm{~m} / \mathrm{s})$ : vehicle (design) speed;

$a\left(\mathrm{~m} / \mathrm{s}^{2}\right)$ : vehicle deceleration rate (e.g., $3.4 \mathrm{~m} / \mathrm{s}^{2}[1]$ and $\left.3.7 \mathrm{~m} / \mathrm{s}^{2}[2]\right)$;

$\mathrm{g}\left(\mathrm{m} / \mathrm{s}^{2}\right)$ : gravitational constant;

$R(\mathrm{~m})$ : horizontal radius;

$e(\% / 100)$ : road cross-slope.

Aiming to quantify the grade effect during the braking process, the laws of mechanics through Eqs. (3) and (4) were applied, assuming time fragments (steps) of $0.01 \mathrm{~s}$, in order to determine both the instantaneous vehicle speed and pure braking distance:

$$
\begin{gathered}
V_{i+1}=V_{i}-\mathrm{g}\left(f_{T}+s\right) t \\
B D_{i}=V_{i} t-\frac{1}{2} \mathrm{~g}\left(f_{T}+s\right) t^{2}
\end{gathered}
$$

where:

$V_{i}(\mathrm{~m} / \mathrm{s})$ : vehicle speed at a specific station $i$;

$V_{i+1}(\mathrm{~m} / \mathrm{s})$ : vehicle speed reduced by the deceleration rate for $t=0.01 \mathrm{~s}$; 
$t(\mathrm{~s})$ : time fragment $(t=0.01 \mathrm{~s})$;

$s(\% / 100)$ : road grade in $i$ position $((+)$ upgrades, $(-)$ downgrades);

$f_{T}$ : friction demand in the longitudinal direction of travel;

$B D_{i}(\mathrm{~m})$ : pure braking distance;

$\mathrm{g}\left(\mathrm{m} / \mathrm{s}^{2}\right)$ : gravitational constant.

By applying Eqs. (3) and (4) subsequently, there is a sequence value $i=k-1$ where $V_{k}$ becomes equal to 0 . The corresponding value of $\sum B D_{k-1}$ represents the total vehicle pure braking distance for the initial value of vehicle speed. The demanded SSD is produced by adding the final pure braking distance to the distance travelled during the driver's perception-reaction time (first component of Eq. (1)) as follows:

$$
S S D_{\text {demanded }}=V_{0} t+\sum B D_{k-1}
$$

where:

$V_{0}(\mathrm{~m} / \mathrm{s})$ : vehicle initial speed;

$t$ (s): driver's perception-reaction time (e.g., $2.5 \mathrm{~s}$ [1] and $2.0 \mathrm{~s}[2])$;

$\sum B D_{k-1}(\mathrm{~m})$ : total vehicle pure braking distance for the initial value of vehicle speed.

Summarizing the demanded SSD determination, Eq. (1) is used, enriched by the longitudinal friction and actual grade value portions, respectively, the effect of which seems to be significant in combined horizontal and crest vertical curved alignments [22].

\subsection{SSD available Calculation}

The available sight distance depends mainly on the alignment configuration. On horizontal tangents, the road user feels comfortable regarding the length of visible roadway ahead, while on curved sections, the driver's available sight distance is reduced. However, the interaction between horizontal and vertical configuration on compound alignments imposes additional sight restrictions, furthermore assisted by various cross-sectional elements, such as the presence of Jersey barriers (on divided highways), metal steel guardrails, or retaining walls, where even more critical safety situations may rise.
In this paper, in order to laterally position the driver's eyes at any desired offset from the typical cross-section centerline, as well as to identify visibility levels due to the presence of these elements, the term "roadlines" is introduced.

Roadlines are defined as lines running longitudinally across the roadway that splits the road into areas of uniform or linearly varied transverse slope. Fig. 3 shows, in cross-sectional view, an example of the required roadlines in order to perform an SSD adequacy examination on the passing lane of a divided road section. In the same figure, it can be seen that six roadlines should be defined per direction of travel, where besides the centerline:

- four roadlines define the Jersey barrier layout (points 1-4);

- one roadline defines the driver's eye at an axis offset equal to half of the passing lane width (point 5);

- one roadline (point 6 ) describes the roadway edge line.

The roadline calculation step is user-specified and delivers a number $n$ of cross-sections where $n$ is defined as the total roadway length divided by the selected calculation step. In general, a step value of $5 \mathrm{~m}$ delivers adequate precision.

The coordination of the roadlines is performed on every cross-section from the very first to $n$, based on a relative coordinate system coinciding on the roadway's centerline and formed by the horizontal $(x)$ and vertical (y) distance of the roadline point (Fig. 3), which is subsequently transformed to the absolute roadway coordinate system.

Furthermore, by connecting a point on one roadline with two relative points on an adjacent roadline, a network of triangles is created representing the roadway surface. Similar is the process for the formation of relevant triangles regarding the rest distinctive parts of a typical cross-section shown in Fig. 2. Between adjacent cross-sections where abrupt roadline variations occur, an extra cross-section is interpolated. For example, in areas of road formation 


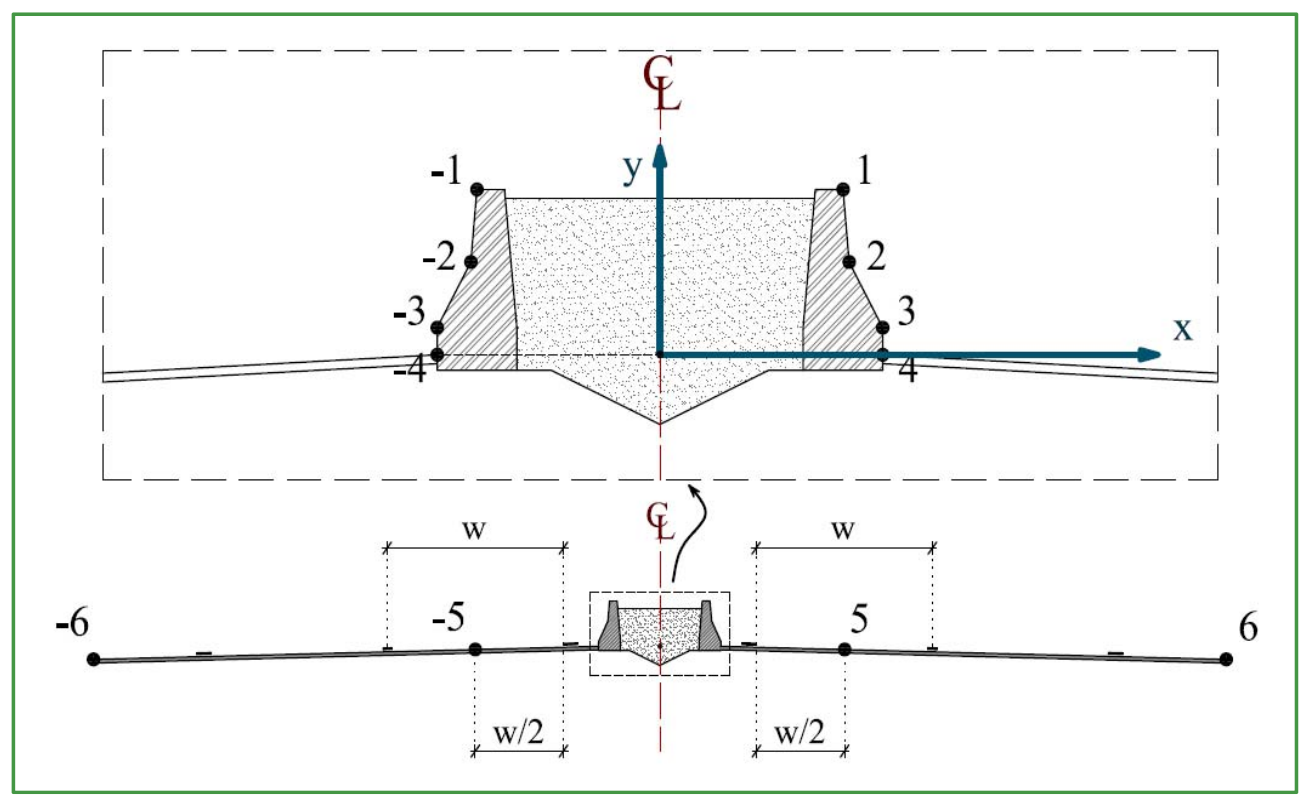

Fig. 3 Roadline layout for SSD adequacy examination on the passing lane of a divided road section (the (-) sign refers to the left roadway; w: width; $\mathbf{E}:$ center line).

transitions between cuts and fills, the extra station is calculated based on the adjacent cut and fill heights.

The creation of the final roadway model is based on the triangulation of the above mentioned distinctive parts. Finally, the road model is superimposed on the ground model (initially triangulated) and the two models are merged with the prevailing road model.

The previously formed triangles are parts of different planes in space, the equations of which can be specified. A plane is defined by three non-linear points in space (which, in this case, are the vertices of the triangle), let points be $\mathrm{A}\left(x_{A}, y_{A}, z_{A}\right), \mathrm{B}\left(x_{B}, y_{B}, z_{B}\right)$ and $\mathrm{C}\left(x_{C}, y_{C}, z_{C}\right)$, and the coordinates of which are known from the triangulation above. Assuming a plane equation:

$$
a x+b y+c z+d=0
$$

where, $a, b, c$ and $d$ are defined as:

$$
\begin{gathered}
a=y_{A}\left(z_{B}-z_{C}\right)+y_{B}\left(z_{C}-z_{A}\right)+y_{C}\left(z_{A}-z_{B}\right) \\
b=z_{A}\left(x_{B}-x_{C}\right)+z_{B}\left(x_{C}-x_{A}\right)+z_{C}\left(x_{A}-x_{B}\right) \\
c=x_{A}\left(y_{B}-y_{C}\right)+x_{B}\left(y_{C}-y_{A}\right)+x_{C}\left(y_{A}-y_{B}\right) \\
d=-x_{A}\left(y_{B} z_{C}-y_{C} z_{B}\right)- \\
x_{B}\left(y_{C} z_{A}-y_{A} z_{C}\right)-x_{C}\left(y_{A} z_{B}-y_{B} z_{A}\right)
\end{gathered}
$$

The driver's line of sight is, on the other hand, a three-dimensional line, which can be defined by two known points in space. In this case, these two points are the driver's eye and obstacle, let points be $E\left(x_{E}, y_{E}, z_{E}\right)$ and $O\left(x_{O}, y_{O}, z_{O}\right)$, respectively. In parametric form, the line's equation is:

$$
P(t)=E+\overrightarrow{E O} t, t \in[0,1]
$$

where:

$P(t)$ : a point on the line;

$E$ : line's initial point $(t=0)$;

$\overrightarrow{E O}$ : direction vector, defined as: $\overrightarrow{E O}=$ $\left(x_{O}-x_{E}, y_{O}-y_{E}, z_{O}-z_{E}\right)$.

By substituting the above in Eq. (11), the following parametric equations are derived:

$$
\begin{gathered}
x=x_{E}+\left(x_{O}-x_{E}\right) t \\
y=y_{E}+\left(y_{O}-y_{E}\right) t \\
z=z_{E}+\left(z_{O}-z_{E}\right) t
\end{gathered}
$$

where, $x, y, z$ : coordinates of a point along the line for a value of the parameter $t$.

Furthermore, the point corresponding to the line and plane intersection, let point be $M\left(x_{M}, y_{M}, z_{M}\right)$ as shown in Fig. 4a, is defined by substituting the line's equations in the plane equation and solving for $t$. In this case, the value of the parameter corresponding to the point of intersection $\left(t_{M}\right)$ is defined as: 


$$
t_{M}=\frac{a x_{O}+b y_{O}+c z_{O}+d}{a\left(x_{O}-x_{E}\right)+b\left(y_{O}-y_{E}\right)+c\left(z_{O}-z_{E}\right)}
$$

Finally, applying Eqs. (12)-(15), the coordinates of the intersection point are defined:

$$
\begin{gathered}
x_{M}=x_{E}+\left(x_{O}-x_{E}\right) t_{M} \\
y_{M}=y_{E}+\left(y_{O}-y_{E}\right) t_{M} \\
z_{M}=z_{E}+\left(z_{O}-z_{E}\right) t_{M}
\end{gathered}
$$

In order to specify whether point $M$ lies inside the triangle area, the following process is utilized: a random line on the $x-y$ projection of the triangle passing through the projection of point $M$ is constructed. Let $A B, B C$ and $A C$ be the points of intersection of this line with the corresponding sides (or their extensions) of the triangle (Fig. 4b). The following quantities are computed:

$$
\begin{aligned}
& k=\left(y_{A B}-y_{A}\right)\left(y_{A B}-y_{B}\right) \\
& l=\left(y_{B C}-y_{B}\right)\left(y_{B C}-y_{C}\right) \\
& m=\left(y_{A C}-y_{A}\right)\left(y_{A C}-y_{C}\right)
\end{aligned}
$$

where, $y_{A B}, y_{B C}$ and $y_{A C}$ are the ordinates of points $A B$, $B C$ and $A C$, respectively.

Four separate cases are distinguished:

- If $k, l, m>0$, then the point lies outside the triangle;

- If $k, l<0$ and $m>0$, then: if $\left(y_{M}-y_{B C}\right)\left(y_{M}-y_{A B}\right)$ $<0$, then the point lies inside the triangle, otherwise, the point lies outside the triangle;

- If $l, m<0$ and $k>0$, then: if $\left(y_{M}-y_{B C}\right)\left(y_{M}-y_{A C}\right)$ $<0$, then the point lies inside the triangle, otherwise, the point lies outside the triangle;

- If $k, m<0$ and $l>0$, then: if $\left(y_{M}-y_{A B}\right)\left(y_{M}-y_{A C}\right)$ $<0$, then the point lies inside the triangle, otherwise, the point lies outside the triangle.

\subsection{SSD Adequacy}

SSD adequacy is granted when:

$$
S S D_{\text {demanded }} \leq S S D_{\text {available }}
$$

The available and demanded SSD values are defined through the difference of the road stations between starting and ending points, assumed at any desired axis offset and equal to the distance between the road's centerline and, usually, half of the examined lane width. The above process, illustrated in the flowchart of Fig. 5, is incorporated in $\mathrm{H} 12$ road design software.

In other words, the applied methodology is based on identifying areas of interrupted vision lines between driver and obstacle being less than the required distance necessary to bring the vehicle to a halt. The precision of the available SSD definition is a function of the selected incremental distance (calculation step) between two sequential points. Test runs performed internally have shown that for a $5 \mathrm{~m}$ calculation step and for approximately $70,000 \sim 80,000$ triangles describing the digital road surface and the terrain, the required SSD adequacy investigation, regarding modern desktop computers, is performed in approximately 10 15 $\mathrm{min}$.

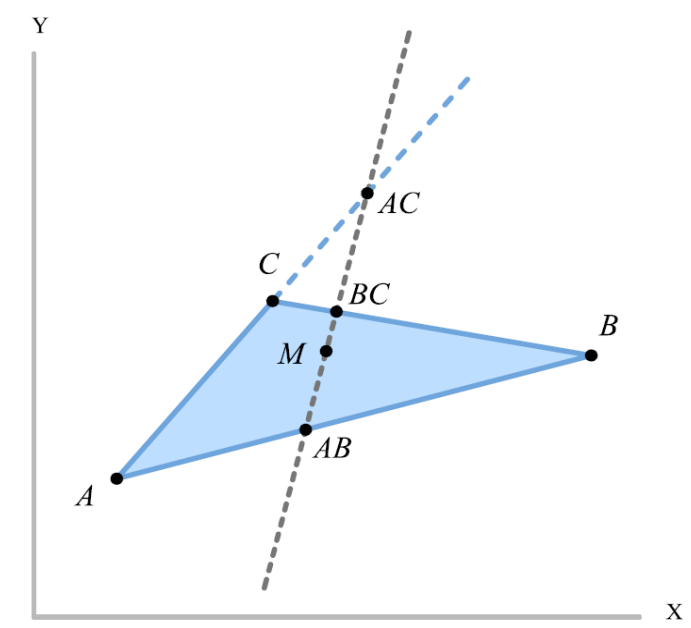

(b)

Fig. 4 (a) Line-plane intersection; (b) intersection point inside triangle boundary. 


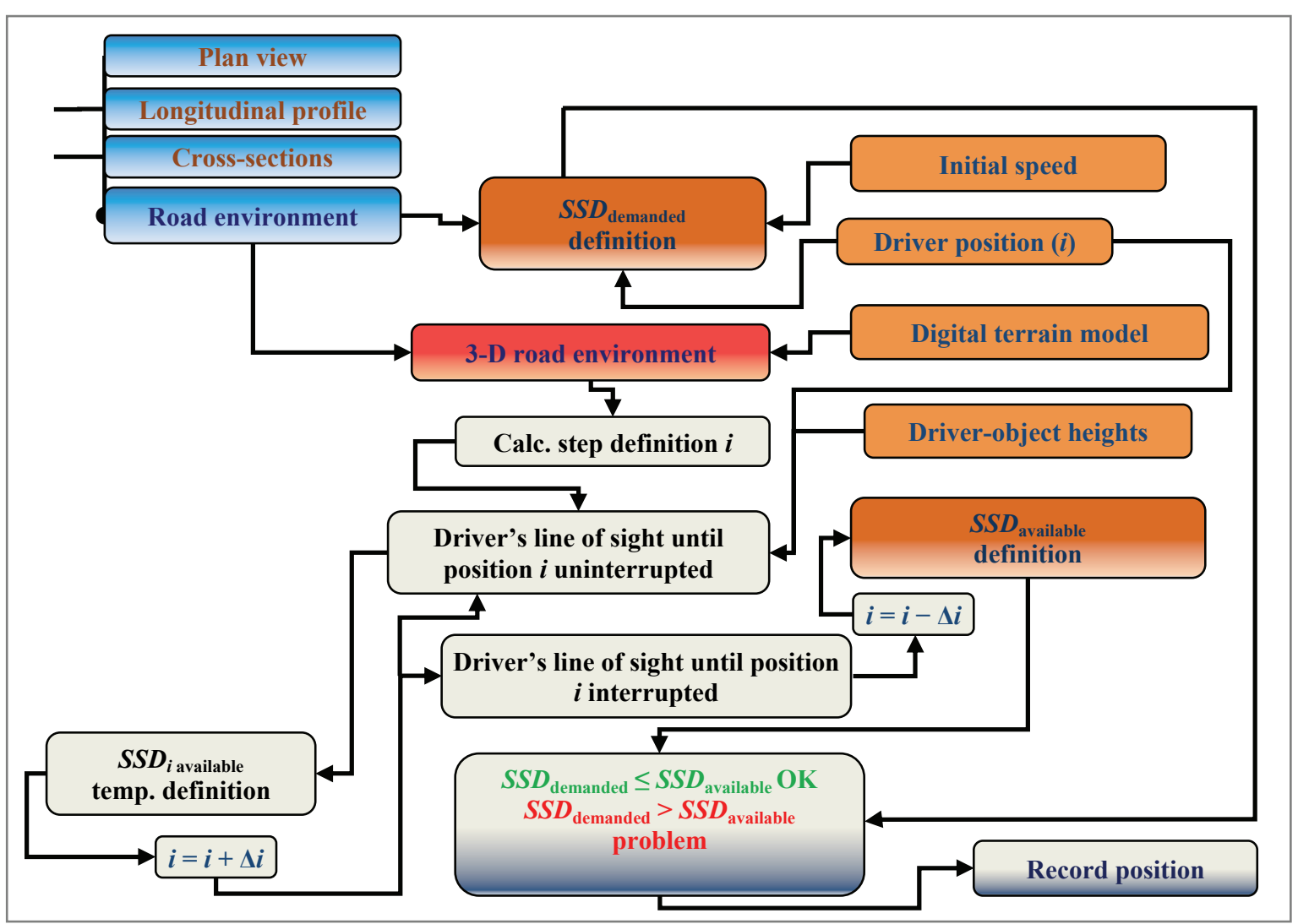

Fig. 5 Flowchart of SSD adequacy investigation.

The credibility of H12 software in defining the $S S D_{\text {available }}$ was validated against an existing road section lacking SSD adequacy, which was surveyed via laser scanner [23]. The available SSD values, extracted graphically utilizing appropriate market software [24], were correlated next to the relevant SSD values extracted from $\mathrm{H} 12$, where a complete match was found.

\section{Case Study}

In order to give a clear view of the H12 software SSD adequacy investigation outputs, the right branch of a hypothetical divided highway section of approximately 4,300 $\mathrm{m}$ was designed according to the German Guidelines [2], assuming $120 \mathrm{~km} / \mathrm{h}$ design speed. The open roadway's cross-section consists of a Jersey barrier, two traffic lanes, the passing lane, which was selected to examine SSD provision, and an emergency lane. Due to the steep landscape, a tunnel of $1,250 \mathrm{~m}$ is proposed between St. $3+000$ and St. $4+250$
(Fig. 6a).

The two utilized cross-sections (open roadway and tunnel) are shown through Fig. $6 \mathrm{~b}$ where certain details found in RAA (German Freeway Design Guidelines) [2] should be emphasized:

- The inner shoulder width was considered $0.75 \mathrm{~m}$ in open roadway areas and $0.50 \mathrm{~m}$ inside tunnels (in the latter case the tunnel inner walls were assumed vertical);

- Driver's eyes height as well as object height was both assumed $1.00 \mathrm{~m}$, offset $1.80 \mathrm{~m}$ laterally from the inner side of the passing lane;

- The Jersey barrier height was set to $0.90 \mathrm{~m}(0.81$ $\mathrm{m}$ plus safety margin), where the curvature at the top increases the inner shoulder by $0.22 \mathrm{~m}$.

Furthermore, assuming that the project specifications impose an advisory speed of $100 \mathrm{~km} / \mathrm{h}$, at the entering portal area, the effective length of the tunnel (length along which the advisory speed of $100 \mathrm{~km} / \mathrm{h}$ is enforced) was inserted as well, consisting of the following: 

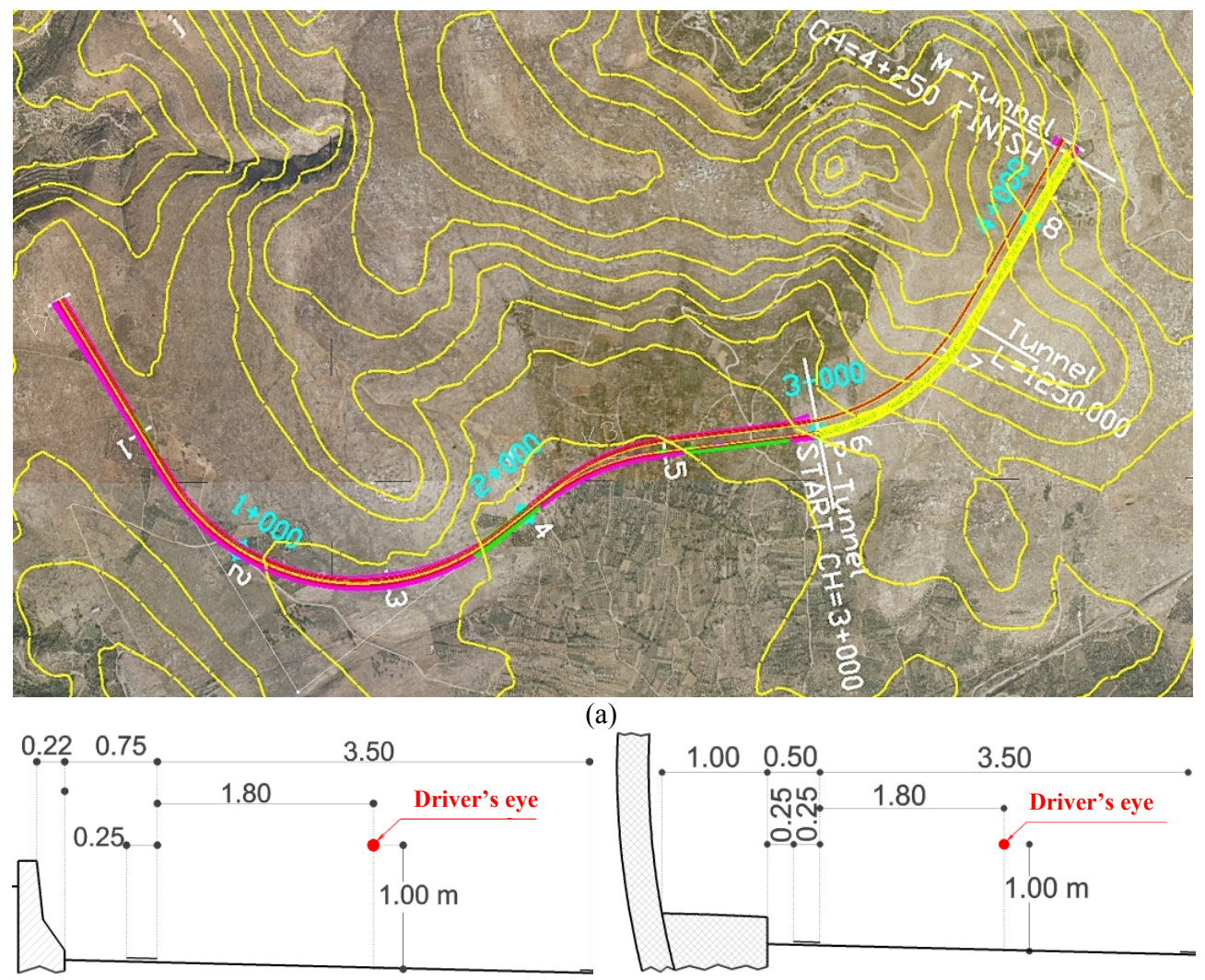

(b)

Fig. 6 (a) Horizontal alignment; (b) cross-sections utilized (units in m).

- $300 \mathrm{~m}$ section in advance of the entering portal;

- extra $200 \mathrm{~m}$ segment ahead as a transition zone where the vehicle speed of $120 \mathrm{~km} / \mathrm{h}$ is transitioned to $100 \mathrm{~km} / \mathrm{h}$.

Finally, since the adopted deceleration rate of 3.7 $\mathrm{m} / \mathrm{s}^{2}$ by RAA [2] corresponds to wet road surface conditions, in order to incorporate realistic emergency braking situations, dry pavement was assumed beyond a $300 \mathrm{~m}$ section inside the tunnel structure where the conservative braking friction value of 0.65 (deceleration $_{\text {dry }}=0.65 \times 9.81 \mathrm{~m} / \mathrm{s}^{2}$ ) was assumed.

The roadway's longitudinal, as well as linearly projected horizontal, profiles are shown in Fig. $7 \mathrm{~b}$. The SSD adequacy investigation (Fig. 7a) is formed by a horizontal and two vertical axes. The horizontal axis represents the road stations where the right vertical axis shows the vehicle's advisory initial speed values along the roadway, based on the above assumptions. The left vertical axis illustrates various SSD values corresponding to the stations of the utilized compound alignment, where three different types of SSDs are shown. The blue line refers to the demanded SSD, where the green line denotes the available SSD, based on roadway's longitudinal profile (2-D assessment).

The objective of the present methodology is not limited in simply defining SSD shortage areas, since a direct overview regarding the design elements that restrict the driver's vision is offered as well. For this reason, the multi-colored 3-D available SSD line, which in general offers less sight distance compared to the relevant 2-D green line, is colored either purple, red, yellow or pink, based on whether the side road formations and/or cut slopes, the roadway surface, the Jersey barrier or the tunnel lateral walls, "hide" the obstacle from the driver, respectively.

A closer look at Fig. 7 reveals two different under design zones, located at the areas where the blue line ( $\left.S S D_{\text {demanded }}\right)$ overlaps the actual available SSD (multi-colored line). In terms of quantifying the SSD safety violation area between the $S S D_{\text {demanded }}$ and the 

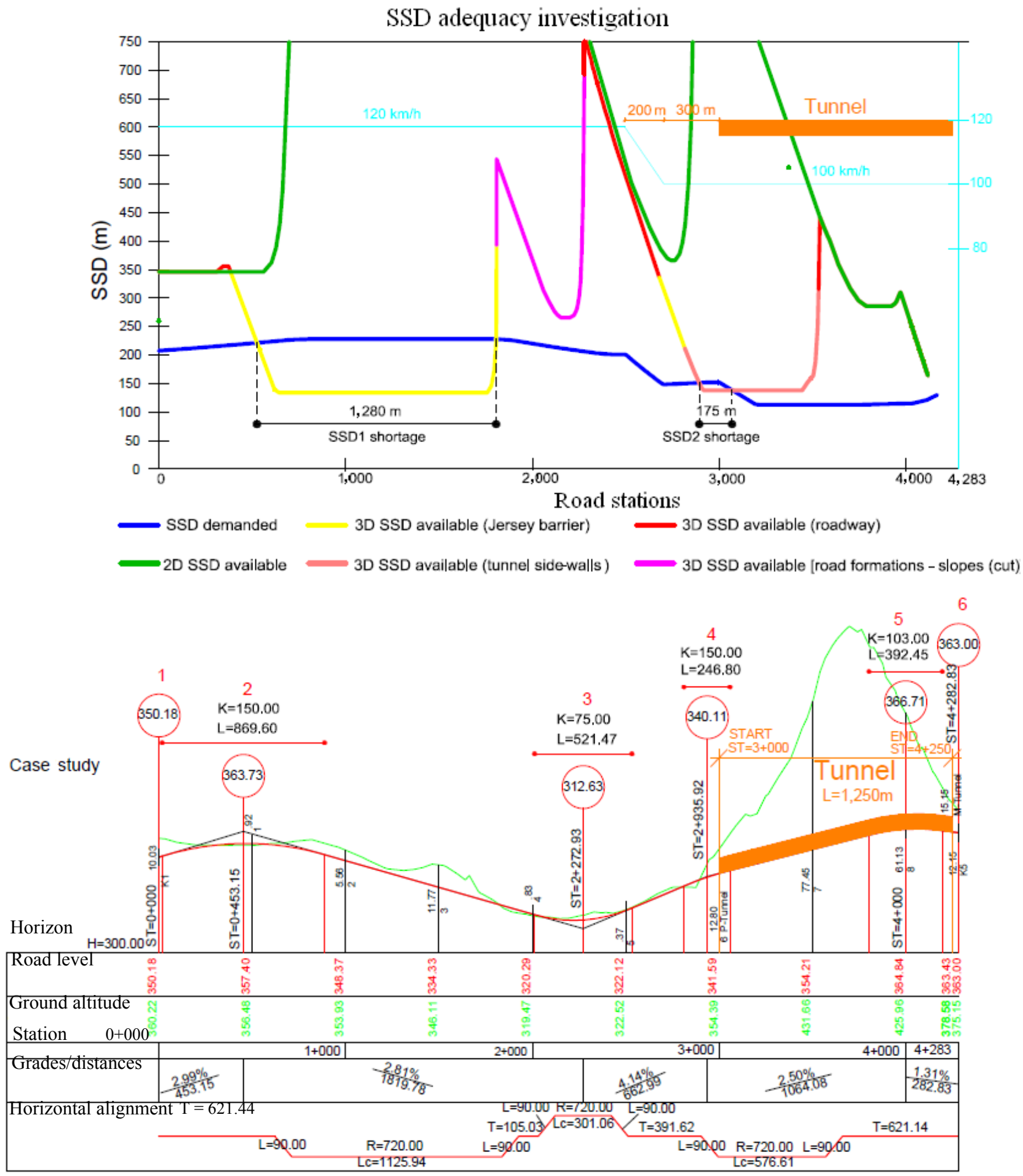

Fig. 7 Roadway's longitudinal profile and SSD adequacy investigation (unit in meters).

$S S D_{\text {available }}(3-\mathrm{D})$, the total SSD1 shortage zone is approximately $1,280 \mathrm{~m}$ (area between St.0+520 and St.1+800), where the relevant SSD2 shortage zone is approximately $175 \mathrm{~m}$ (area between St.2+895 and
St.3+070). As a result, in the above unsuccessful horizontal-vertical arrangement, the Jersey barrier and the tunnel lateral walls are responsible for the delivered SSD1 and SSD2 shortage areas, respectively. 


\section{Conclusions}

The present paper describes a SSD adequacy investigation resulting from any combination of design elements and based on the difference between the available and the demanded SSD. $S S D_{\text {demanded, on one }}$ hand, is defined by utilizing the point mass model, introduced by many design guidelines worldwide, and enriched by the actual values of grade and friction variation due to the effect of vertical curves and vehicle cornering, respectively. On the other hand, the $S S D_{\text {available }}$ is described as the driver's line of sight towards the object height at a certain offset in 3-D roadway environment.

The proposed methodology can accurately perform SSD adequacy investigation in any 3-D road environment given that the ground, road and roadside elements are inserted. SSD shortage issues are created in roadway areas where the vision lines between driver and obstacle are less than the relevant demanded distances that safely route a vehicle to stop.

Furthermore, H12 software provides flexibility among every road design and/or vehicle dynamic parameter inserted (e.g., lateral positioning of both driver and obstacle, vehicle speed and friction variations (tunnel areas of freeways where pavement is considered dry)) as well as direct overview regarding design elements responsible for SSD inadequacies. As a result, precious guidance is provided to the designer for further alignment improvement but mostly an accurate aid to implement geometric design control criteria with respect to both existing and new road sections is delivered.

The present software is, nowadays, used for educational purposes in undergraduate classes at the Department of Transportation Planning and Engineering of Civil Engineering School in NTUA, where regarding the preparation of diploma theses, it is considered to be an essential tool. Many highway design and consultant firms in Greece use it as well.

However, it is important to underline the fact that the parameters used in the present paper (speed values, perception of reaction time, etc.) refer to daylight driving conditions, as, on the one hand, the vehicle speed values in night time driving conditions are 6 15 $\mathrm{km} / \mathrm{h}$ less [25] and, on the other hand, the road view geometry changes.

Finally, it should not be ignored that the human factor, including perception-reaction procedure and the friction reserve utilized in the lateral direction during the braking process, might impose additional restrictions and, consequently, influence the braking process to some extent.

\section{References}

[1] AASHTO (American Association of State Highway and Transportation Officials). 2011. A Policy on Geometric Design of Highways and Streets. Washington, DC: AASHTO.

[2] German Road and Transportation Research Association, Committee, Geometric Design Standards. 2008. Guidelines for the Design of Freeways. Germany: RAA.

[3] Ministeriode Fomento (Ministeriode Development). 2001. Instrucción de Carreteras, Norma 3.1-IC "Trazado" (Spanish Road Design Guidelines). Spain: Ministeriode Development.

[4] Ministry of Environment, Regional Planning and Public Works. 2001. Guidelines for the Design of Road Projects, Part 3, Alignment (OMOE-X (Greek Road Design Guidelines)). Greece: Ministry of Environment, Regional Planning and Public Works.

[5] NTUA. 2012. H12, Road Design Software. Greece: NTUA.

[6] Hassan, Y., Easa, S. M., and Abd El Halim, A. O. 1997. "Design Considerations for Combined Highway Alignments." Journal of Transportation Engineering 123 (1): $60-8$.

[7] Sanchez, E. 1994. "Three-Dimensional Analysis of Sight Distance on Interchange Connectors." In Transportation Research Record 1445, 101-8.

[8] Hassan, Y., Easa, S. M., and Abd El Halim, A. O. 1996. "Analytical Model for Sight Distance Analysis on Three-Dimensional Highway Alignments." Transportation Research Record 1523 (1): 1-10.

[9] Lovell, D. J., Jong, J. C., and Chang, P. C. 2001. "Improvement to the Sight Distance Algorithm." Journal of Transportation Engineering 127 (4): 283-8.

[10] Nehate, G., and Rys, M. 2006. "3-D Calculation of Stopping-Sight Distance from GPS Data." Journal of Transportation Engineering 132 (6): 691-8. 
[11] DiVito, M., and Cantisani, G. 2010. "D.I.T.S.: A Software for Sight Distance Verification and Optical Defectiveness Recognition." Presented at the 4th International Symposium on Highway Geometric Design, TRB (Transportation Research Board), Valencia, Spain.

[12] García, A. 2004. "Optimal Vertical Alignment Analysis for Highway Design-Discussion.” Journal of Transportation Engineering 130 (1): 138.

[13] Ismail, K., and Sayed, T. 2007. "New Algorithm for Calculating 3-D Available Sight Distance.” Journal of Transportation Engineering 133 (10): 572-81.

[14] Chou, A. M., Perez, V., Garcia, A., and Rojas, M. 2010. "Optimal 3-D Coordination to Maximize the Available Stopping Sight Distance in Two-Lane Roads.” Presented at the 4th International Symposium on Highway Geometric Design, TRB, Valencia, Spain.

[15] Romero, M. A., and García, A. 2007. "Optimal Overlapping of Horizontal and Vertical Curves Maximizing Sight Distance by Genetic Algorithms." Presented at the 86th Annual Meeting of the Transportation Research Board, Washington, DC.

[16] Yan, X., Radwan, E., Zhang, F., and Parker, J. C. 2008. "Evaluation of Dynamic Passing Sight Distance Problem Using a Finite-Element Model." Journal of Transportation Engineering 134 (6): 225-35.

[17] Zimmermann, M. 2005. "Increased Safety Resulting from Quantitative Evaluation of Sight Distances and Visibility Conditions of Two-Lane Rural Roads." Presented at the 3rd International Symposium on Highway Geometric Design, Chicago, USA.

[18] Kim, D., and Lovell, D. 2010. “A Procedure for 3-D Sight
Distance Evaluation Using Thin Plate Splines.” Presented at the 4th International Symposium on Highway Geometric Design, TRB, Valencia, Spain.

[19] Jha, M., Karri, G. A. K., and Kuhn, W. 2011. “A New 3-Dimensional Highway Design Methodology for Sight Distance Measurement." Presented at the 90th Annual Meeting of the Transportation Research Board, Washington, DC.

[20] Mertzanis, F., and Hatzi, V. 2011. "Model for Sight Distance Calculation and Three-Dimensional Alignment Evaluation in Divided and Undivided Highways." Presented at the 3rd International Conference on Road Safety and Simulation, Indianapolis, USA.

[21] Krempel, G. 1965. "Experimenteller Beitrag zu Untersuchungen an Kraftfahrzeugreifen (Experimental Contribution to the Studies of Motor Vehicle Tires)." Dissertation, TU Karlsruhe. (in German)

[22] Mavromatis, S., Palaskas, S., and Psarianos, B. 2012. "Continuous Three-Dimensional Stopping Sight Distance Control on Crest Vertical Curves." Advances in Transportation Studies 28: 17-30.

[23] Mavromatis, S., Pagounis, V., Palaskas, S., and Maroudas, D. 2009. "Stopping Sight Distance Assessment via 3-D Road Scanning." Presented at the 4th National Conference in Road Safety, Athens, Greece.

[24] Leica. 2008. Leica Cyclone-3-D Point Cloud Processing Software. Germany: Leica Inc.

[25] Malakatas, K. 2012. "Operating Speed Predicting Model on Two-Lane Rural Roads during Night-Time." Diploma thesis, National Technical University of Athens. 